\title{
The Interaction of Air Pollutant Molecules with Germanene and Silicene: a Density Functional Theory Study
}

\author{
Muhammad Rifqi Al Fauzan ${ }^{1 *}$, Wijayanti Dwi Astuti ${ }^{1}$, Ghorby Al Fauzan ${ }^{1}$, Sholihun $^{2}$ \\ ${ }^{1}$ Department of Electrical Engineering and Informatics, Vocational College, Universitas Gadjah Mada, \\ Yogyakarta, Indonesia. \\ ${ }^{2}$ Department of Physics, Faculty of Mathematics and Natural Sciences, Universitas Gadjah Mada, \\ Yogyakarta, Indonesia. \\ *email: alfauzan.rifqi@ugm.ac.id
}

Received February 19, 2018; Accepted May 4, 2018; Available online May 31, 2018

\begin{abstract}
We investigate the adsorption of atmospheric pollutants on germanene and silicene using density functional theory (DFT) calculations. In this study, we use carbon monoxide (CO) and nitric oxide (NO) as the pollutant molecules. Electronic properties of germanene and silicene are explored to obtain a good understanding in the adsorption process. Our calculation results show both germanene and silicene provide an identical mechanism of adsorption. Germanene and silicene adsorb $\mathrm{CO}$ and NO spontaneously with physisorption and chemisorption types for $\mathrm{CO}$ and $\mathrm{NO}$ molecules, respectively. We also conduct the Mulliken charges calculations to evaluate the transformation of atomic charges due to the adsorption process. From Mulliken charge calculation results we can confirm the existence of charge transfer between the adsorbent materials and the pollutant molecules. This phenomenon lies behind the occurrence of the adsorption process.
\end{abstract}

Keywords: DFT, germanene, interaction, pollutant molecules, silicene

\section{INTRODUCTION}

An atmospheric pollutant is a hazardous substance in the air that has harmful effects on humans and the ecosystems. Carbon monoxide (CO) and nitric oxide (NO) are two of the most dangerous gases in our atmosphere, it can cause various health effect from dizziness to death (Ischiropoulus et al., 1996; Raub et al., 2000). Both CO and NO gases are available abundantly in the atmosphere since they are produced by vehicles and industrial exhaust gases. By this fact, it is clear that we need to find material that has the ability to adsorb pollutant gases such as $\mathrm{CO}$ and NO. In this study, we compare the adsorption ability of germanene and silicene with $\mathrm{CO}$ and $\mathrm{NO}$ molecules as adsorbed materials. Germanene and silicene are analog to graphene but they use germanium and silicon atoms instead. Since graphene exhibit excellence sensitivity to its chemical environment (Schedin et al., 2007), germanene and silicene are expected to show similar result or even better. Some previous theoretical studies have reported the ability of germanene and silicene to adsorb various gas molecules by using density functional theory. $\mathrm{Hu}$ et al. (2014) reported that $\mathrm{NH}_{3}, \mathrm{NO}$, and $\mathrm{NO}_{2}$ chemically adsorbed on silicene. Another calculation conducted by $\mathrm{Xia}$ et al showed that $\mathrm{N}_{2}, \mathrm{CO}, \mathrm{CO}_{2}$ are physically adsorbed while $\mathrm{NH}_{3}, \mathrm{NO}, \mathrm{NO}_{2}, \mathrm{O}_{2}$ are chemically adsorbed on germanene (Xia et al., 2014). In our study, we did calculations in order to understand the mechanism of adsorpsion of $\mathrm{CO}$ and $\mathrm{NO}$ on both silicene and germanene. We investigated the charge difference distribution and then calculated the charge per atom before and after adsorption process.

On the experimental studies, germanene was successfully grown on $\operatorname{Pt}(111)$ and $\mathrm{Au}$ (111) (Li et al., 2014; Dávila et al., 2014), as well as silicene on $\mathrm{Ag}(111), \mathrm{ZrB}_{2}(0001)$, and $\operatorname{Ir}(111)$ surfaces (Jamgotchian et al., 2012; Fleurence et al., 2012; Meng et al., 2013).

\section{EXPERIMENTAL SECTION}

\section{Material and Methods}

We carried out the first-principles electronics-structure calculations by means of density functional theory (DFT) to find out the possibility of using germanene and silicene as adsorbent material for $\mathrm{CO}$ and $\mathrm{NO}$ molecules through the adsorption mechanism. In this study, we use a $4 \times 4 \times 1$ supercell of each germanene and silicene which contains 32 atoms with the concentration of $\mathrm{CO}$ and $\mathrm{NO}$ about $6.25 \%$. All calculations in this research were performed using Open Source Package for Material Explorer (OpenMX) code (Ozaki, 
2003) with Generalized-Gradient Approximation (GGA) with Perdew-BurkeErnzerhof (PBE) (Perdew et al, 1996) as the exchange-correlation form. OpenMX is based on the norm-conserving pseudopotentials (Troullier and Martins, 1991), the wave functions were expanded by a linear combination of multiple pseudo-atomic orbitals (LCPAO) (Ozaki, 2003; Ozaki and Kino, 2004). A $10 \times 10 \times 1$ k-points in Brillouin zone is used for electronic structure calculation to gain the desired result, energy convergence criteria are adjusted at $10^{-5}$ Hartree. Large enough vacuum space in the $\mathrm{z}$ direction is used to prevent interactions between adjacent slabs for both germanene and silicene.

\section{RESULTS AND DISCUSSION}

\section{Geometrical Properties}

In the geometry optimization calculations, we found that lattice constant of germanene and silicene are about $4.053 \AA$ and $3.890 \AA$, respectively. These results seem in a good agreement with other works reported by Acun et al. (2015) and Gimbert et al. (2014). After the most stable geometries of are obtained, then we introduce $\mathrm{CO}$ and $\mathrm{NO}$ molecules on both germanene and silicene surfaces to evaluate the formation energy of each system. The stable positions of CO and NO molecules on both adsorbent surfaces are

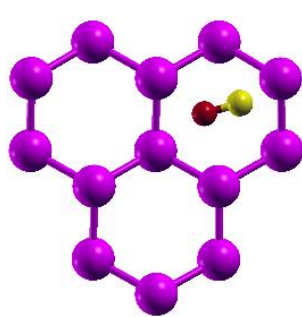

(a)

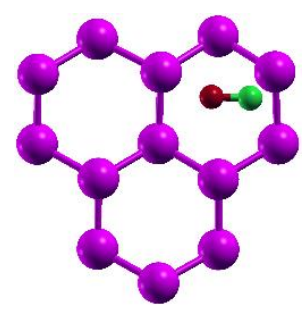

(b)

shown in Figure 1. Then, the formation energy $\left(E_{\text {form }}\right)$ is obtained by simple arithmetic formulation as defined in Equation 1:

$E_{\text {form }}=E_{\text {molecule } / \text { adsorbent }}-\left(E_{\text {molecule }}+\right.$

$\left.E_{\text {adsorbent }}\right)$

Where $E_{\text {molecule }}, E_{\text {adsorbent }}$, and $E_{\text {molecule/adsorbent }}$ are the total energy of the molecule, pristine adsorbent, and gas molecule adsorbed on adsorbent material, respectively. We have four different systems, they are $\mathrm{CO}$ adsorbed on germanene, $\mathrm{NO}$ adsorbed on germanene, $\mathrm{CO}$ adsorbed on silicene, and $\mathrm{NO}$ adsorbed on silicene. For simplification, we call them with $\mathrm{CO} /$ germanene, $\mathrm{NO} /$ germanene, $\mathrm{CO} /$ silicene, and $\mathrm{NO}$ /silicene, serially. By using Equation 1, we get formation energy of each system. Our results are listed in Table 1.

The adsorption processes that occur on all of our systems are spontaneous reactions since they exhibit negative values on their formation energies. It means the adsorption processes can be done without any external energy needed. This becomes an essential requirement for every adsorbent material. Although both germanene and silicene yield same negative values of formation energies, there is a notable difference happen. Our calculation result as listed in Table $\mathbf{1}$ shows that silicene provide higher formation energy compared to silicene. It means adsorption and also desorption processes on silicene is easier to happen than germanene.

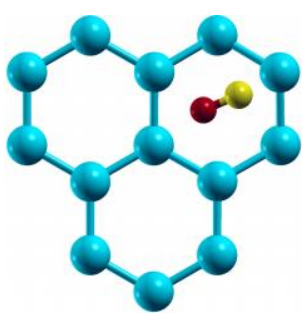

(c)

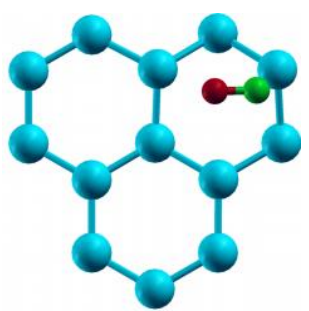

(d)

Figure 1. The most stable positions of the pollutant molecules on various systems: (a) $\mathrm{CO} /$ germanene, (b) NO/germanene, (c) CO/silicene, and (d) NO/silicene. The purple, cyan, red, yellow, and green balls are germanium, silicon, oxygen, carbon, and nitrogen atoms, respectively.

Table 1. Formation energies of various systems are presented in $\mathrm{eV}$ units, types of adsorption are also shown.

\begin{tabular}{ccc}
\hline System & Formation energy $(\mathrm{eV})$ & Type of adsorption \\
\hline CO/germanene & -0.558 & Physisorption \\
NO/germanene & -1.141 & Chemisorption \\
CO/silicene & -0.005 & Physisorption \\
NO/silicene & -0.022 & Chemisorption \\
\hline
\end{tabular}


Therefore from the formation energy point of view, we can sum up that both germanene and silicene have the ability to adsorb $\mathrm{CO}$ and $\mathrm{NO}$ molecules with spontaneous reactions, but it is more likely to happen in silicene than in germanene.

\section{Electronic Properties}

The charges of the total density of states (DOS) were analyzed to determine the type of adsorption in each system, as plotted in Figure 2. The electronic properties of germanene are semi-metal with zero-gap at the Dirac point as well as silicene. Both germanene and silicene as adsorbent materials exhibit identical result in reacting to the presence of $\mathrm{CO}$ and $\mathrm{NO}$ molecules. Adsorption of $\mathrm{CO}$ molecule does not change the electronic properties of adsorbent materials (see Figure 2 (c) and (d)). Therefore adsorption of $\mathrm{CO}$ is categorized as physisorption process through van der Waals interaction between adsorbent materials and gas molecules. On the contrary, the NO adsorption is classified as chemisorption process since it is able to open band gap of adsorbent materials about $0.18 \mathrm{eV}$. So the electronic properties of the material are no longer semi-metal but turned into the semiconductor with narrow $0.18 \mathrm{eV}$ band gap. The covalent bonds occur in $\mathrm{Ge}_{5}-\mathrm{N}$ and $\mathrm{Si}_{5}-\mathrm{N}$ (see Figure 3 (b) and (d)) with bond length about $2,2 \AA$ and $1,9 \AA$, respectively. In addition, this result also tells us about the possibility of using germanene and silicene as sensor materials application for NO molecule.
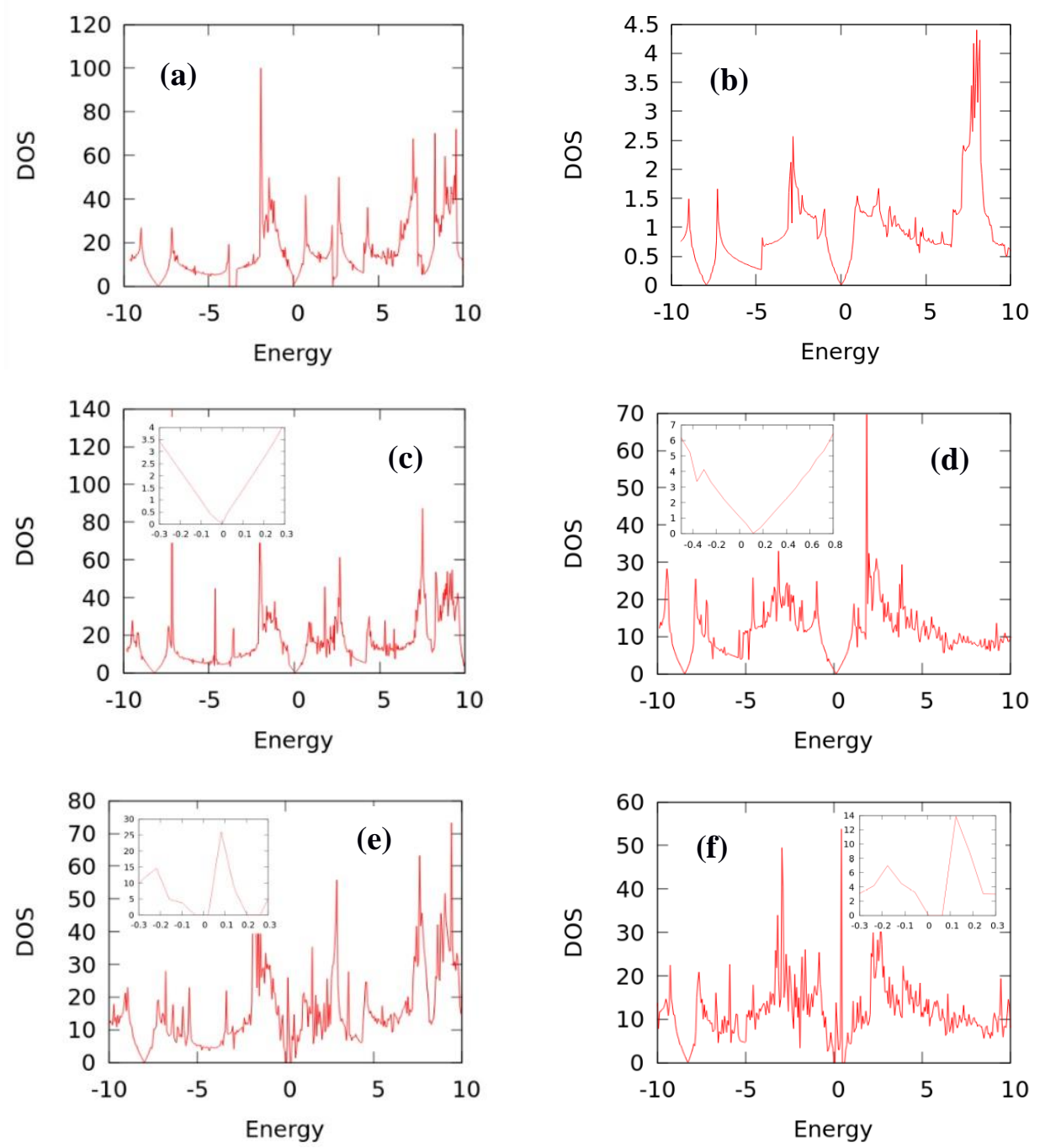

Figure 2. Density of states (DOS) of (a) pristine germanene (b) pristine silicene (c) CO/germanene (d) $\mathrm{CO} /$ silicene (e) $\mathrm{NO} /$ germanene (f) $\mathrm{NO} /$ silicene systems. These figures represent the electronic properties of each system. 


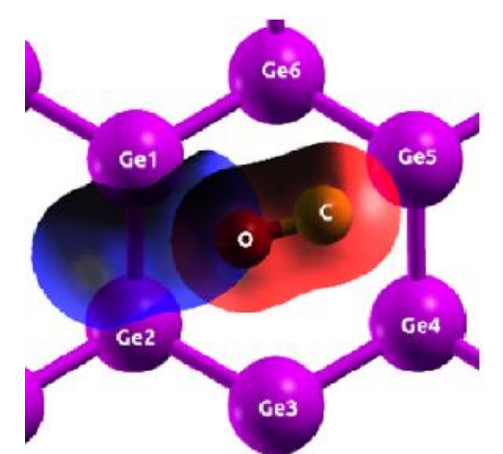

(a)

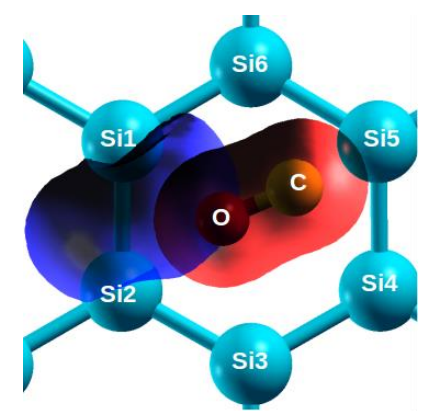

(c)

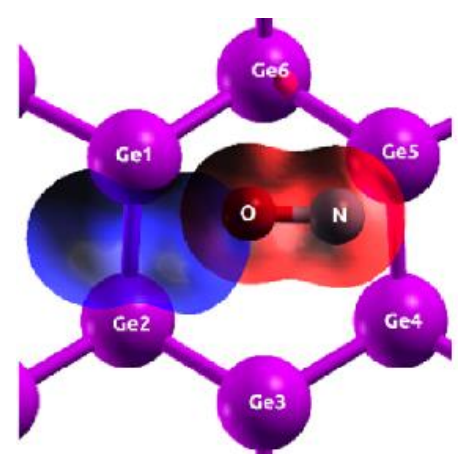

(b)

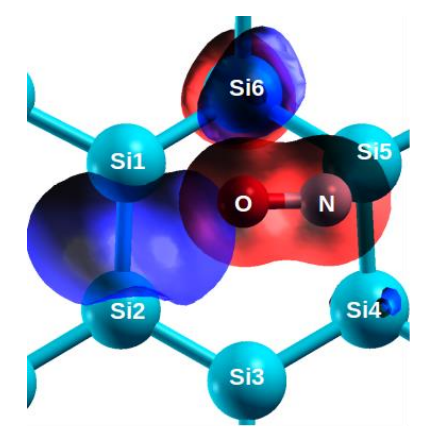

(d)

Figure 3. Charge difference distribution of (a) $\mathrm{CO} /$ germanene (b) $\mathrm{NO} /$ germanene (c) $\mathrm{CO} /$ silicene and (d) NO/silicene systems. The red and blue regions indicate the positive and negative values. Electron charges flowed from blue region to red region. Only pollutant molecules and its neighboring atoms of adsorbent materials are shown.

Table 2. Atomic charges of pollutant molecules and neighboring atoms of adsorbent materials based on Mulliken charge analysis.

\begin{tabular}{|c|c|c|c|}
\hline \multirow{2}{*}{ Atom } & \multirow{2}{*}{ Before adsorption } & \multicolumn{2}{|c|}{ After adsorption } \\
\hline & & CO/germanene & NO/germanene \\
\hline Ge1 & 4.000 & 3.961 & 3.922 \\
\hline $\mathrm{Ge} 2$ & 4.000 & 4.004 & 4.011 \\
\hline $\mathrm{Ge} 3$ & 4.000 & 3.981 & 3.952 \\
\hline $\mathrm{Ge} 4$ & 4.000 & 4.026 & 3.987 \\
\hline Ge5 & 4.000 & 3.981 & 3.848 \\
\hline Ge6 & 4.000 & 4.030 & 4.043 \\
\hline O_CO/germanene & 6.341 & 6.370 & - \\
\hline C_CO/germanene & 3.659 & 3.640 & - \\
\hline O_NO/germanene & 6.211 & - & 6.268 \\
\hline $\mathrm{N} \_$NO/germanene & 4.790 & - & 5.004 \\
\hline Si1 & 4.000 & 3.991 & 3.996 \\
\hline $\mathrm{Si} 2$ & 4.000 & 3.972 & 4.011 \\
\hline $\mathrm{Si} 3$ & 4.000 & 3.979 & 3.942 \\
\hline $\mathrm{Si} 4$ & 4.000 & 3.990 & 3.988 \\
\hline $\mathrm{Si} 5$ & 4.000 & 3.988 & 3.770 \\
\hline Si6 & 4.000 & 3.988 & 3.969 \\
\hline O_CO/silicene & 6.341 & 6.369 & - \\
\hline C_CO/silicene & 3.659 & 3.660 & - \\
\hline O_NO/silicene & 6.211 & - & 6.261 \\
\hline $\mathrm{N} \_$NO/silicene & 4.790 & - & 5.042 \\
\hline
\end{tabular}


The mechanism of adsorption process can be explained by investigating the interaction between pollutant molecules and adsorbent materials. We explore this by considering the existence of charge transfer between pollutant molecules and adjacent atoms of adsorbent materials. The present of charge transfer can be verified through calculation of charge difference distribution $(\Delta \rho)$ as defined in Equation 2.

$$
\Delta \rho=\rho_{\text {molecule } / \text { adsorbent }}-\left(\rho_{\text {adsorbent }}+\right.
$$

Where

$\rho_{\text {molecule }}$,

$\rho_{\text {adsorbent }}$, $\rho_{\text {molecule/adsorbent }}$ are the charge density of the pollutant molecule, adsorbent material, and pollutant molecule adsorbed on adsorbent material, respectively. The results are illustrated in Figure 3. The accumulation and the loss of charge are depicted by positive and negative values of the charge difference distribution, respectively. This result confirms the presence of charge transfer between adsorbent materials and pollutant molecules.

In order to acquire a better understanding of the adsorption processes, we did Mulliken charge analysis. The charge around each atom is calculated by adding population over $s p$ orbital. Its results are presented in Table 2.

Figure 3 shows the presence of charge accumulation around each pollutant in all systems. These are consistent with the results provide by Mulliken charge analysis as shown in Table 2. The charge of $\mathrm{CO}$ and $\mathrm{NO}$ molecules are increased due to the adsorption processes, $\mathrm{CO}$ molecule receives additional charge from germanene by $0.010 e$ and from silicene by $0.029 e$. While NO molecule receives $0.271 e$ and $0.303 e$ from germanene and silicene surfaces, respectively.

\section{CONCLUSIONS}

We carried out first-principles calculations by means of density functional theory (DFT) to investigate the interactions between pollutant molecules and adsorbent materials. In this research, we use $\mathrm{CO}$ and $\mathrm{NO}$ as pollutant molecules, while for adsorbent material we use germanene and silicene. Our calculation results show that both germanene and silicene exhibit identical adsorption processes in response to the presence of $\mathrm{CO}$ and $\mathrm{NO}$ molecules. The mechanism of interactions between pollutant molecules and adsorbent materials can be evaluated by investigating the charge difference distributions and charge transfer between them. By using Mulliken charge analysis we successfully confirm the existence of charge transfer from adsorbent materials to pollutant molecules. The total charge of $\mathrm{CO}$ molecule is increased by $0.010 e$ after adsorbed on germanene and by $0.029 e$ after adsorbed on silicene. While the charge of NO molecule increases about $0.271 e$ and $0.303 e$ after adsorbed on germanene and silicene, respectively.

\section{ACKNOWLEDGMENTS}

This work was funded by Peningkatan Kapasitas Peneliti Dosen Muda Universitas Gadjah Mada project under contract No. 376/DIT.LIT/2017. The calculations were performed on the high-performance computing facilities (DSSDI) at Universitas Gadjah Mada.

\section{REFERENCES}

Acun, A., Zhang, L., Bampoulis, P., Farmanbar, M., van Houselt, A., Rudenko, A. N., Lingenfelder, M., Brocks, G., Poelsema, B., Katsnelson, M. I., and Zandvliet, H. J. W. (2015). Germanene: the germanium analogue of graphene. Journal of Physics: Condensed Matter, 27(44), 443002443014.

Cahangirov, S., Topsakal, M., Aktürk, E., Şahin, H., and Ciraci, S. (2009). Twoand one-dimensional honeycomb structures of silicon and germanium. Physical Review Letters, 102(23), 236804-236808.

Dávila, M. E., Xian, L., Cahangirov, S., Rubio, A., and Lay, G. L. (2014). Germanene: a novel two-dimensional germanium allotrope akin to graphene and silicene. New Journal of Physics, 16(9), 095002095008.

Fleurence, A., Friedlein, R., Ozaki, T., Kawai, H., Wang, Y., and Yamada-Takamura, Y. (2012). Experimental Evidence for Epitaxial Silicene on Diboride Thin Films. Physical Review Letters, 108(24), 245501-245506.

Gimbert, F., Lee, C-. C., Friedlein, R., Fleurence, A., Yamada-Takamura, Y., Ozaki, T. (2014). Diverse forms of bonding in two-dimensional $\mathrm{Si}$ allotropes: Nematic orbitals in the MoS2 
structure. Physical Review B, 90(16), 165423-165428.

Hu, W., Xia, N., Wu, X., Li, Z., and Yang, J. (2014). Silicene as a highly sensitive molecule sensor for $\mathrm{NH}_{3}, \mathrm{NO}$, and $\mathrm{NO}_{2}$. Physical Chemistry Chemical Physics, 16, 6957-6962.

Ischiropoulus, H., Beers, M. F., Ohnishi, S. T., Fisher, D., Garner, S. E., and Thom, S. R. (1996). Nitric oxide production and perivascular nitration in brain after carbon monoxide poisoning in the rat. The Journal of Clinical Investagion, 97(10), 2260-2267.

Jamgotchian, H., Colignon, Y., Hamzaoui, N., Ealet, B., Hoarau, J. Y., Aufray, B., and Bibeérian, J. P. (2012). Growth of silicene layers on $\operatorname{Ag}(111)$ : unexpected effect of the substrate temperature. Journal of Physics: Condensed Matter, 24(17), 172001.

Kara, A., Enriquez, H., Seitsonend, A. P., Voone, L. C. L. Y., Vizzini, S., Aufrayg, B., and Oughaddoub, H. (2012). A review on silicene-new candidate for electronics. Surface Science Reports, 67(1), 1-18.

Li, L., Lu, S.-Z., Pan, J., Qin, Z., Wang, Y.-Q., Wang, Y., Cao, C.-Y., Du, S., and Gao, H.-J. (2014). Buckled Germanene Formation on $\mathrm{Pt}(111)$. Advanced Materials, 26(28), 4820-4824.

Meng, L., Wang, Y., Zhang, L., Du, S., Wu, R., Li, L., Zhang, Y., Li, G., Zhou, H., Hofer, W. A., Gao, H.-G. (2013). Buckled silicene formation on $\operatorname{Ir}(111)$. Nano Letters, 13(2), 685-690.
Ozaki, T. (2003). Variationally optimized atomic orbital for large-scale electronic structures. Physical Review B, 67(15), 155108-155113.

Ozaki, T. and Kino, H. (2004). Numerical atomic basis orbital from $\mathrm{H}$ to $\mathrm{Kr}$. Physical Review B, 69(19), 195113195132.

Perdew, J. P., Burke, K., and Ernzerhof, M. (1996). Generalized gradient approximation made simple. Physical Review Letters, 77(18), 3865-3868.

Raub, J. A., Nolf, M.-M., Hampson, N. B., Thom, S. R. (2000). Carbon monoxide poisoning - a public health perspective. Toxicology, 145 (1), 1-14.

Scalise, E., Houssa, M., van den Broek, B., Afanas'ev, V. V., and Stesmans, A. (2013) Vibrational properties of silicene and germanene. Nano Research, 6(1), 19-28.

Schedin, F., Geim, A. K., Morozov, S. V., Hill, E. W., Blake, P., Katsnelson, M. I., and Novoselov, K. S. (2007). Detection of individual gas molecules adsorbed on graphene. Nature Materials, 6 (9), 652655.

Troullier, N. and Martins, J. L. (1991). Efficient pseudopotentials for planewave calculations. Physical Review B, 43(3), 1993-2006.

Xia, W., Hu, W., Li, Z., and Yang, J. (2014). A first-principles study of gas adsorption on germanene. Physical Chemistry Chemical Physics, 16, 22495-22498. 